\title{
ON PERIODIC ORBITS IN DISCRETE-TIME CASCADE SYSTEMS
}

HUIMIN LI AND XIAO-SONG YANG

Received 28 March 2006; Accepted 19 July 2006

We present some results on existence, minimum period, number of periodic orbits, and stability of periodic orbits in discrete-time cascade systems. Some examples are presented to illustrate these results.

Copyright (c) 2006 H. Li and X.-S. Yang. This is an open access article distributed under the Creative Commons Attribution License, which permits unrestricted use, distribution, and reproduction in any medium, provided the original work is properly cited.

\section{Introduction}

Consider the following discrete-time cascade systems:

$$
\begin{gathered}
x(n+1)=f(x(n), y(n)), \\
y(n+1)=g(y(n)),
\end{gathered}
$$

where $x \in \mathbb{R}^{n}, y \in \mathbb{R}^{m}, f: \mathbb{R}^{n} \times \mathbb{R}^{m} \rightarrow \mathbb{R}^{n}$ is a continuous function and $g: \mathbb{R}^{m} \rightarrow \mathbb{R}^{m}$ is a function.

An interesting problem we are concerned with is the following question.

Question. Does system (1.1) have periodic orbits when system (1.2) has periodic orbits?

Recently, there have been a lot of researches in the literature on the periodicity of discrete-time dynamical systems $[1,3-8,10,11]$. However, to the authors' knowledge the above question has not received investigations, therefore in this paper we study the above question and obtain a fundamental result. On the basis of the fundamental result in Section 2 we further discuss the number and the minimum period of periodic orbits of system (1.1) when system (1.2) has periodic orbits. In Section 3, we present a result on the stability of periodic orbits.

\section{Results on existence, minimum period, and number of periodic orbits}

In this section, we present some results on the existence, the minimum period, and the number of periodic orbits. 
Before stating our results, we first recall a fixed point theorem due to Brower, which is useful in the following arguments.

Theorem 2.1 (Brower's fixed point theorem). Let $F: C \subset \mathbb{R}^{n} \rightarrow C \subset \mathbb{R}^{n}$ be a continuous mapping, where $C$ is a nonempty, bounded, close, and convex set, then $F$ has a fixed point.

Its proof can be found in [2].

Moreover we make the following assumptions for system (1.1).

(H1) When $y \neq 0, f(0, y) \neq 0$.

(H2) $\varlimsup_{\lim _{\|x\| \rightarrow \infty}}(\|f(x, y)\| /\|x\|)=\mu(y)<1$, where $\mu: \mathbb{R}^{m} \rightarrow \mathbb{R}$ is a function, $\|\cdot\|$ denotes the Euclidean norm.

Now we state our results. First we give the following theorem (Theorem 2.2), and it is addressed by the similar technique from [9, Theorem 2.2].

Theorem 2.2. Suppose that (H1) and (H2) hold. If system (1.2) has a nontrivial periodic orbit, then system (1.1) has a corresponding nontrivial periodic orbit.

Proof. Because system (1.2) has a nontrivial periodic orbit, without loss of generality we assume that the periodic orbit is $o\left(y_{0}\right)=\left\{y_{0}, y_{1}, \ldots, y_{k-1}\right\}$ with minimum period $k$, where $k$ is a positive integer, $y_{0}=g\left(y_{k-1}\right)$, and $y_{i+1}=g\left(y_{i}\right), i=0,1, \ldots, k-2$.

Since condition (H2) holds, for every $y_{i}, i=0,1, \ldots, k-1$, there exists a corresponding $m_{i}, i=0,1, \ldots, k-1$, such that when $\|x\|>m_{i},\left\|f\left(x, y_{i}\right)\right\|<\|x\|$.

Letting $\overline{m_{i}}=\max _{\|x\| \leq m_{i}}\left\|f\left(x, y_{i}\right)\right\|>0, M_{i}=\max \left(m_{i}, \overline{m_{i}}\right), M=\max \left\{M_{i}, i=0,1, \ldots\right.$, $k-1\}>0$, one has that if $\|x\| \leq M$, then for every $y_{i}, i=0,1, \ldots, k-1,\left\|f\left(x, y_{i}\right)\right\| \leq M$, which implies that the set $U=\{x \mid\|x\| \leq M\}$ is a positive invariant set of the following systems:

$$
x(n+1)=f\left(x(n), y_{i}\right), \quad i=0,1, \ldots, k-1 .
$$

Letting $f_{i}=f\left(x, y_{i}\right), i=0,1, \ldots, k-1, F=f_{k-1}, \ldots, f_{0}$, we easily have that $F: U \rightarrow U$ is a continuous mapping. Then by Brower's fixed point theorem one has that the function $F$ has a fixed point $\bar{x}_{0}$, that is, $F\left(\bar{x}_{0}\right)=\bar{x}_{0}, f_{k-1}, \ldots, f_{0}\left(\bar{x}_{0}\right)=\bar{x}_{0}$.

Letting $\bar{x}_{i}=f_{i-1}\left(\bar{x}_{i-1}\right)$, we get $f_{k-1}\left(\bar{x}_{k-1}\right)=\bar{x}_{0}$, thus the orbit $o\left(\bar{x}_{0}\right)=\left\{\bar{x}_{0}, \bar{x}_{1}, \ldots, \bar{x}_{k-1}\right\}$ is a periodic orbit. This implies that $o\left(\bar{x}_{0}\right)$ contains a periodic orbit whose minimum period $\bar{k}$ divides $k$, where $\bar{k}$ is a positive integer.

In addition, according to condition (H1), we have that there exists $i_{0} \in\{0,1, \ldots, k-1\}$ such that $\bar{x}_{i_{0}} \neq 0$. This shows that $o\left(\bar{x}_{0}\right)$ contains a nontrivial periodic orbit. The proof is complete.

It seems that the period of $o\left(\bar{x}_{0}\right)$ should not be less than that of system (1.2), however the following example shows that this is not always the case.

Now we apply Theorem 2.2 to discuss the following example.

Example 2.3.

$$
\begin{gathered}
x(n+1)=f(x(n), y(n)), \\
y(n+1)=g(y(n)),
\end{gathered}
$$


where

$$
\begin{aligned}
& x \in \mathbb{R}, y \in \mathbb{R}, \quad f(x, y)= \begin{cases}\frac{x}{2}+1, & x \in(-\infty,+\infty), y \in(-\infty, 0.6), \\
\frac{x}{2}+1+(y-0.6)(y-1.1018), & x \in(-\infty,+\infty), y \in[0.6,+\infty),\end{cases} \\
& g(y)=3.4986 y(1-y) .
\end{aligned}
$$

By numerical analysis we easily see that system (2.3) has a periodic orbit $\{0.5000$, $0.8746,0.3836,0.8272\}$. According to Theorem 2.2, one has that system (2.2) has a periodic orbit, and the periodic orbit is $\{1.9168,1.9584\}$.

Remark 2.4. In Example 2.3, we have the following facts.

(a) The system $x(n+1)=f(x(n), 0)=(1 / 2) x(n)+1$ is globally asymptotically stable which implies that the system does not have nontrivial periodic orbits. However its perturbed system has a nontrivial periodic orbit.

(b) The function $f(x, y)$ satisfies the following conditions: $f\left(x, y_{0}\right)=f\left(x, y_{2}\right), f(x$, $\left.y_{1}\right)=f\left(x, y_{3}\right)$, where $y_{0}=0.5000, y_{1}=0.8746, y_{2}=0.3836$, and $y_{3}=0.8272$.

(c) The minimum period of the periodic orbit of system (2.3) is 4 , but the minimum period of its corresponding periodic orbit in system (2.2) is 2, which is a little surprising.

Considering the fact (c), we give the following theorem (Theorem 2.5). And the following additional condition is assumed for system (1.1).

(H3) When $y^{\prime} \neq y^{\prime \prime}, f\left(x, y^{\prime}\right) \neq f\left(x, y^{\prime \prime}\right)$.

Theorem 2.5. Suppose that (H1), (H2), and (H3) hold. If system (1.2) has a periodic orbit of minimum period $k$, then the minimum period of its corresponding periodic orbit in system (1.1) is also $k$.

Proof. In terms of the proof of the above theorem, we have that system (1.1) has a periodic orbit $o\left(\bar{x}_{0}\right)=\left\{\bar{x}_{0}, \bar{x}_{1}, \ldots, \bar{x}_{j-1}\right\}$ of minimum period $j$, where $j$ divides $k$ and $j \leq k$. Below we prove that the minimum period of the above periodic orbit is $k$, that is, $j=k$. To complete our proof we assume that $j<k$. Since $o\left(\bar{x}_{0}\right)=\left\{\bar{x}_{0}, \bar{x}_{1}, \ldots, \bar{x}_{j-1}\right\}$ is a periodic orbit, it is evident that $\bar{x}_{0}=\bar{x}_{j}, \bar{x}_{1}=\bar{x}_{j+1}$.

Because $\bar{x}_{0}=\bar{x}_{j}$, by condition (H3) we have $f\left(\bar{x}_{0}, y_{0}\right) \neq f\left(\bar{x}_{j}, y_{j}\right)$, that is, $\bar{x}_{1} \neq \bar{x}_{j+1}$ which is a contradiction to the above equality. Therefore the minimum period of the above periodic orbit is also $k$. The proof is complete.

Remark 2.6. Consider the following system.

Example 2.7.

$$
\begin{gathered}
x(n+1)=f(x(n), y(n)), \\
y(n+1)=g(y(n)),
\end{gathered}
$$


where

$$
x \in \mathbb{R}, y \in \mathbb{R}, \quad f(x, y)=\frac{y}{1+|y|}(1+x), \quad g(y)= \begin{cases}1, & y=\frac{1}{2} \\ \frac{1}{2}, & y=1 \\ y+1, & \text { other. }\end{cases}
$$

It is easy to see that system (2.6) only has a periodic orbit $\{1 / 2,1\}$ with minimum period 2 and in Example 2.7 the conditions stated in Theorem 2.2 are satisfied. Thus by Theorem 2.2 we have that system $(2.5)$ has a periodic orbit $\{4 / 5,3 / 5\}$ whose minimum period is the same as the minimum period of $\{1 / 2,1\}$.

Theorem 2.8. Suppose that (H1), (H2), and (H3) hold. If system (1.2) has $m$ different periodic orbits, then system (1.1) also has $m$ different periodic orbits at least.

To prove this theorem, we establish the following elementary lemma.

Lemma 2.9. Suppose that $o\left(c_{0}\right)=\left\{c_{0}, c_{1}, \ldots, c_{k-1}\right\}$ and $o\left(d_{0}\right)=\left\{d_{0}, d_{1}, \ldots, d_{l-1}\right\}$ are two different periodic orbits of minimum period $k$, $l$ of system (1.2), where $k$ and l are two positive integers, then $c_{i} \neq d_{j}, i=0,1, \ldots, k-1, j=0,1, \ldots, l-1$.

This lemma is easy to be proved, and its proof is omitted here.

Proof of Theorem 2.8. Assuming that $o\left(c_{0}\right)=\left\{c_{0}, c_{1}, \ldots, c_{k-1}\right\}$ and $o\left(d_{0}\right)=\left\{d_{0}, d_{1}, \ldots, d_{l-1}\right\}$ are two different periodic orbits of minimum period $k, l$ of system (1.2), by Lemma 2.9 we have $c_{i} \neq d_{j}, i=0,1, \ldots, k-1, j=0,1, \ldots, l-1$, and by Theorem 2.5 we have that system (1.1) has two corresponding periodic orbits $o\left(c_{0}^{\prime}\right)=\left\{c_{0}^{\prime}, c_{1}^{\prime}, \ldots, c_{k-1}^{\prime}\right\}$ and $o\left(d_{0}^{\prime}\right)=$ $\left\{d_{0}^{\prime}, d_{1}^{\prime}, \ldots, d_{l-1}^{\prime}\right\}$ whose minimum periods are, respectively, $k$ and $j$.

Below we want to prove that the above orbits are different. For this purpose, we assume that the two periodic orbits $o\left(c_{0}^{\prime}\right)=\left\{c_{0}^{\prime}, c_{1}^{\prime}, \ldots, c_{k-1}^{\prime}\right\}$ and $o\left(d_{0}^{\prime}\right)=\left\{d_{0}^{\prime}, d_{1}^{\prime}, \ldots, d_{l-1}^{\prime}\right\}$ are the same, thus there exist $\bar{i} \in\{0,1, \ldots, k-1\}$ and $\bar{j} \in\{0,1, \ldots, k-1\}$ such that $c_{\bar{i}}^{\prime}=d_{\bar{j}}^{\prime}$ which implies that $c_{\bar{i}+1}^{\prime}=d_{\bar{j}+1}^{\prime}$. In addition, according to $c_{\bar{i}+1}^{\prime}=f\left(c_{\bar{i}}^{\prime}, c_{\bar{i}}\right), d_{\bar{j}+1}^{\prime}=f^{\prime}\left(d_{j}^{\prime}, d_{\bar{j}}\right)$, and condition (H3), one has $c_{\bar{i}+1}^{\prime} \neq d_{\bar{j}+1}^{\prime}$, which contradicts the equality $c_{i+1}^{\prime}=d_{\bar{j}+1}^{\prime}$.

Consequently $o\left(c_{0}^{\prime}\right)=\left\{c_{0}^{\prime}, c_{1}^{\prime}, \ldots, c_{k-1}^{\prime}\right\}$ and $o\left(d_{0}^{\prime}\right)=\left\{d_{0}^{\prime}, d_{1}^{\prime}, \ldots, d_{l-1}^{\prime}\right\}$ are two different periodic orbits. Therefore by the above result and Theorem 2.5, we conclude that system (1.1) has $m$ different periodic orbits at least.

Remark 2.10. Consider the following system.

Example 2.11.

$$
\begin{gathered}
x(n+1)=f(x(n), y(n)), \\
y(n+1)=g(y(n)),
\end{gathered}
$$


where

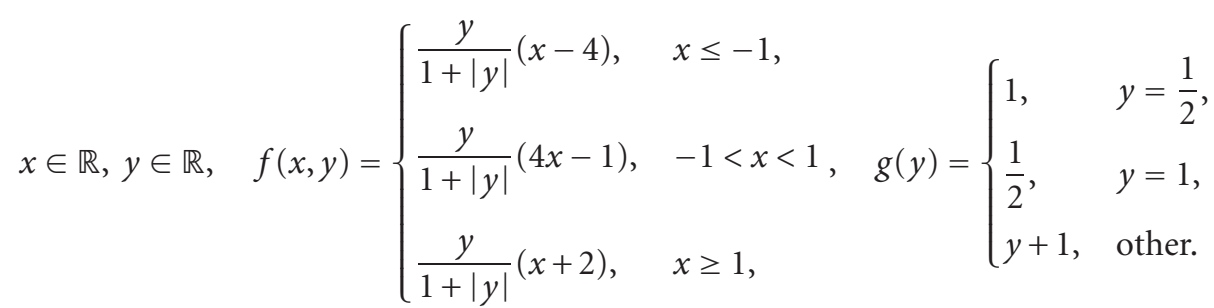

System (2.9) in this example has only a periodic orbit $\{1 / 2,1\}$, and the conditions stated in Theorem 2.8 are satisfied in this example. However, system (2.8) has the following three periodic orbits: $\{-12 / 5,-16 / 5\},\{7 / 10,3 / 5\}$, and $\{8 / 5,6 / 5\}$.

This example shows that for a periodic orbit of system (1.2), the number of its corresponding periodic orbits of system (1.1) may be greater than one.

Below we give a more precise estimate on the number of periodic orbits. First, we need the following additional condition for system (1.1).

(H4)

$$
\left\|f\left(x_{1}, y\right)-f\left(x_{2}, y\right)\right\|<\left\|x_{1}-x_{2}\right\|, \quad \forall x_{1}, x_{2} \in \mathbb{R}^{n}, y \in \mathbb{R}^{m}
$$

Theorem 2.12. Suppose that all conditions stated in Theorem 2.8 are satisfied and (H4) holds. Then system (1.1) only has $m$ different periodic orbits.

Proof. In terms of the proof of Theorem 2.2, we know that $F=f_{k-1}, \ldots, f_{0}$ has a fixed point. That is,

$$
f_{k-1}, \ldots, f_{0}\left(\bar{x}_{0}\right)=\bar{x}_{0}
$$

According to condition (H4), it is easy to prove that the fixed point is unique. This implies that when system (1.2) has a periodic orbit, system (1.1) has a corresponding periodic orbit that is unique.

Therefore by the above result, Theorem 2.8, and its proof, we have that when system (1.2) has $m$ different periodic orbits, system (1.1) has only $m$ different periodic orbits.

The proof is complete.

Remark 2.13. Consider the following system.

Example 2.14.

$$
\begin{gathered}
x(n+1)=f(x(n), y(n)), \\
y(n+1)=g(y(n)),
\end{gathered}
$$


where

$$
x \in \mathbb{R}, y \in \mathbb{R}, \quad f(x, y)=\frac{y}{1+|y|}(1+x), \quad g(y)= \begin{cases}y-2, & y<0, y \neq-\frac{1}{2},-1, \\ -1, & y=-\frac{1}{2}, \\ -\frac{1}{2}, & y=-1, \\ y+2, & y \geq 0, y \neq \frac{1}{2}, 1,2, \\ \frac{1}{2}, & y=2, \\ 1, & y=\frac{1}{2}, \\ 2, & y=1 .\end{cases}
$$

It is evident that system (2.14) has the following two periodic orbits: $\{-1,-1 / 2\}$ and $\{1 / 2,1,2\}$. And it is easy to verify that conditions stated in Theorem 2.12 are satisfied in the above example. Then the periodic orbit of system (2.13) that is corresponding to the periodic orbit $\{-1,-1 / 2\}$ is $\{-1 / 5,-2 / 5\}$. The corresponding periodic orbit of $\{1 / 2,1,2\}$ is $\{5 / 4,3 / 4,7 / 8\}$.

\section{A result on stability of periodic orbits}

In view of the results obtained above, we can see that under the conditions ( $\mathrm{H} 1)$ and (H2) for a periodic orbit $o\left(y_{0}\right)=\left\{y_{0}, y_{1}, \ldots, y_{k-1}\right\}$ with minimum period $k$ of system (1.2), there is a corresponding periodic orbit $o\left(x_{0}\right)=\left\{x_{0}, x_{1}, \ldots, x_{k-1}\right\}$ with period $k$ in system (1.1).

In this section, our purpose is to study the stability of the periodic orbit $o\left(z_{0}\right)$, where $o\left(z_{0}\right)=o\left(\left(\begin{array}{l}x_{0} \\ y_{0}\end{array}\right)\right)=\left\{\left(\begin{array}{l}x_{0} \\ y_{0}\end{array}\right),\left(\begin{array}{l}x_{1} \\ y_{1}\end{array}\right), \ldots,\left(\begin{array}{l}x_{k-1} \\ y_{k-1}\end{array}\right)\right\}$. For this purpose, we give the following conditions.

(H5) For each $i=0,1, \ldots, k-1,\left\|f\left(x^{\prime}, y\right)-f\left(x^{\prime \prime}, y\right)\right\| \leq \alpha(y)\left\|x^{\prime}-x^{\prime \prime}\right\|$, where $x^{\prime}$ and $x^{\prime \prime}$ belong to certain neighborhood $U_{i}=B\left(x_{i}, r\right)$ of $x_{i} \in \mathbb{R}^{n}, B\left(x_{i}, r\right)=\left\{x \mid\left\|x-x_{i}\right\|\right.$ $<r\}, y \in \mathbb{R}^{m}$, the function $\alpha: \mathbb{R}^{m} \rightarrow \mathbb{R}^{+}$is a continuous function with $\alpha\left(y_{i}\right)<1$ for $y_{i} \in o\left(y_{0}\right)$, and $\mathbb{R}^{+}$denotes all nonnegative real numbers.

(H6) For each $i=0,1, \ldots, k-1,\left\|f\left(x_{i}, y^{\prime}\right)-f\left(x_{i}, y^{\prime \prime}\right)\right\| \leq \beta\left(x_{i}\right)\left\|y^{\prime}-y^{\prime \prime}\right\|$, where $y^{\prime}$ and $y^{\prime \prime}$ belong to certain neighborhood $V_{i}$ of $y_{i} \in \mathbb{R}^{m}, x_{i} \in o\left(x_{0}\right)$, and $\beta: \mathbb{R}^{n} \rightarrow \mathbb{R}^{+}$is a function.

Before stating our result, we review a definition.

Definition 3.1. An orbit $\left\{x_{n}\right\}(n=0,1, \ldots)$ with the initial value $x_{0}=x$ is said to be stable if it satisfies the following conditions.

For every $\varepsilon>0$, there exists $\delta>0$ such that if $\|x-y\|<\delta$, then $\left\|x_{n}-y_{n}\right\|<\varepsilon$ for every orbit $\left\{y_{n}\right\}$ with the initial value $y$. 
Theorem 3.2. Suppose that all conditions stated in Theorem 2.2 are satisfied, and (H5) and (H6) hold. If a periodic orbit $o\left(y_{0}\right)=\left\{y_{0}, y_{1}, \ldots, y_{k-1}\right\}$ with minimum period $k$ of system (1.2) is stable, then the periodic orbit $o\left(z_{0}\right)$ is also stable, where $o\left(z_{0}\right)=o\left(\left(\begin{array}{l}x_{0} \\ y_{0}\end{array}\right)\right)=$ $\left\{\left(\begin{array}{l}x_{0} \\ y_{0}\end{array}\right),\left(\begin{array}{l}x_{1} \\ y_{1}\end{array}\right), \ldots,\left(\begin{array}{l}x_{k-1} \\ y_{k-1}\end{array}\right)\right\}$, the periodic orbit $o\left(x_{0}\right)=\left\{x_{0}, x_{1}, \ldots, x_{k-1}\right\}$ is corresponding to the periodic orbit $o\left(y_{0}\right)$.

Proof. According to Theorem 2.2, we have that for a periodic orbit $o\left(y_{0}\right)$ with minimum period $k$ of system (1.2), there is a corresponding periodic orbit $o\left(x_{0}\right)$ with period $k$ in system (1.1), therefore the minimum period of $o\left(z_{0}\right)$ is also $k$.

To prove stability of $o\left(z_{0}\right)$, we first give the following key inequalities ((3.1) and (3.2)).

The inequality (3.1) is easily obtained from the conditions (H5) and (H6):

$$
\begin{aligned}
\left\|x_{n+1}^{\prime}-x_{n+1}\right\| & =\left\|f\left(x_{n}^{\prime}, y_{n}^{\prime}\right)-f\left(x_{n}, y_{n}\right)\right\| \leq\left\|f\left(x_{n}^{\prime}, y_{n}^{\prime}\right)-f\left(x_{n}, y_{n}^{\prime}\right)+f\left(x_{n}, y_{n}^{\prime}\right)-f\left(x_{n}, y_{n}\right)\right\| \\
& \leq \alpha\left(y_{n}^{\prime}\right)\left\|x_{n}^{\prime}-x_{n}\right\|+\beta\left(x_{n}\right)\left\|y_{n}^{\prime}-y_{n}\right\|, \quad n \geq 0 .
\end{aligned}
$$

Since $\alpha: \mathbb{R}^{m} \rightarrow \mathbb{R}^{+}$is a continuous function and $\alpha\left(y_{i}\right)<1, i=0,1, \ldots, k-1$, we have that for $y_{i} \in o\left(y_{0}\right)$, there exist $\eta_{i}>0$ such that if $\left\|y-y_{i}\right\|<\eta_{i}$, then $\alpha(y)<\left(1+\alpha\left(y_{i}\right)\right) / 2<1$, here we make $\eta_{i}$ be sufficiently small such that $B\left(y_{i}, \eta_{i}\right) \subset V_{i}$. And we set $\eta=\min _{0 \leq i \leq k-1} \eta_{i}$, by the stability of $o\left(y_{0}\right)$, we have that there exists $0<\delta_{2}^{\prime}<\eta$ such that if $\left\|y_{0}^{\prime}-y_{0}\right\|<\delta_{2}^{\prime}$, then $\left\|y_{n}^{\prime}-y_{n}\right\|<\eta$, for $y_{n} \in o\left(y_{0}\right)$ and $n \geq 0$.

Letting $a=\max _{0 \leq i \leq k-1}\left\{\left(1+\alpha\left(y_{i}\right)\right) / 2\right\}<1$ and $b=\max _{0 \leq i \leq k-1}\left\{\beta\left(x_{i}\right)\right\}$, by the stability of $o\left(y_{0}\right)$, we have that for every $\varepsilon_{1}>0$, there exists $\delta_{2}^{\prime \prime}>0$ such that if $\left\|y_{0}^{\prime}-y_{0}\right\|<\delta_{2}^{\prime \prime}$, then $\left\|y_{n}^{\prime}-y_{n}\right\|<((1-a) / b) \varepsilon_{1}, n \geq 0$. then

Then when letting $\delta_{2}=\min \left(\delta_{2}^{\prime}, \delta_{2}^{\prime \prime}\right)$, we have that for every $\varepsilon_{1}>0$, if $\left\|y_{0}^{\prime}-y_{0}\right\|<\delta_{2}$,

$$
\alpha\left(y_{n}^{\prime}\right)<\frac{1+\alpha\left(y_{n}\right)}{2}<1, \quad\left\|y_{n}^{\prime}-y_{n}\right\|<\frac{1-a}{b} \varepsilon_{1}, \quad \text { for } n \geq 0
$$

Below we use mathematical induction to prove the following result.

For every $\varepsilon_{1}>0$, there exist $\delta_{1}>0$ and $\delta_{2}>0$ such that if $\left\|x_{0}^{\prime}-x_{0}\right\|<\delta_{1}$ and $\left\|y_{0}^{\prime}-y_{0}\right\|<$ $\delta_{2}$, then $\left\|x_{n}^{\prime}-x_{n}\right\|<\varepsilon_{1}, n \geq 1$, where $z_{0}^{\prime}=\left(\begin{array}{l}x_{0}^{\prime} \\ y_{0}^{\prime}\end{array}\right)$ and $z_{0}=\left(\begin{array}{l}x_{0} \\ y_{0}\end{array}\right)$ are the initial values of the orbits $\left\{z_{n}^{\prime}\right\}=\left\{\left(\begin{array}{c}x_{n}^{\prime} \\ y_{n}^{\prime}\end{array}\right)\right\}$ and $\left\{z_{n}\right\}=\left\{\left(\begin{array}{l}x_{n} \\ y_{n}\end{array}\right)\right\}=o\left(z_{0}\right)$, respectively.

Now for every $0<\varepsilon_{1}<r$ when choosing $\delta_{1}=\varepsilon_{1}, \delta_{2}=\min \left(\delta_{2}^{\prime}, \delta_{2}^{\prime \prime}\right)$, by (3.1) and (3.2) we have that

$$
\begin{aligned}
\left\|x_{1}^{\prime}-x_{1}\right\| & \leq \alpha\left(y_{0}^{\prime}\right)\left\|x_{0}^{\prime}-x_{0}\right\|+\beta\left(x_{0}\right)\left\|y_{0}^{\prime}-y_{0}\right\|<a\left\|x_{0}^{\prime}-x_{0}\right\|+b\left\|y_{0}^{\prime}-y_{0}\right\| \\
& <a \varepsilon_{1}+b \frac{1-a}{b} \varepsilon_{1}=\varepsilon_{1} .
\end{aligned}
$$

By mathematical induction, we assume $\left\|x_{n}^{\prime}-x_{n}\right\|<\varepsilon_{1}$. Here our purpose is to prove that $\left\|x_{n+1}^{\prime}-x_{n+1}\right\|<\varepsilon$, by (3.1) we have

$$
\left\|x_{n+1}^{\prime}-x_{n+1}\right\| \leq \alpha\left(y_{n}^{\prime}\right)\left\|x_{n}^{\prime}-x_{n}\right\|+\beta\left(x_{n}\right)\left\|y_{n}^{\prime}-y_{n}\right\| .
$$


8 On periodic orbits in discrete-time cascade systems

By (3.2) we have that

$$
\begin{aligned}
\left\|x_{n+1}^{\prime}-x_{n+1}\right\| & \leq \alpha\left(y_{n}^{\prime}\right)\left\|x_{n}^{\prime}-x_{n}\right\|+\beta\left(x_{n}\right)\left\|y_{n}^{\prime}-y_{n}\right\| \\
& \leq a\left\|x_{n}^{\prime}-x_{n}\right\|+b\left\|y_{n}^{\prime}-y_{n}\right\|<a \varepsilon_{1}+b \frac{1-a}{b} \varepsilon_{1}=\varepsilon_{1} .
\end{aligned}
$$

Therefore by mathematical induction, we have proven that for every $0<\varepsilon_{1}<r$, there exist $\delta_{1}>0$ and $\delta_{2}>0$ such that if $\left\|x_{0}^{\prime}-x_{0}\right\|<\delta_{1}$ and $\left\|y_{0}^{\prime}-y_{0}\right\|<\delta_{2}$, then $\left\|x_{n}^{\prime}-x_{n}\right\|<\varepsilon_{1}$ and $\left\|y_{n}^{\prime}-y_{n}\right\|<((1-a) / b) \varepsilon_{1}$, for $n \geq 1$.

In terms of the above result, for every $0<\varepsilon<((1+a-b) / b) r$, if we choose $0<\varepsilon_{1}=$ $(b /(1+a-b)) \varepsilon<r$, then it is easy to prove that there exists $\delta>0$ such that if $\left\|z_{0}^{\prime}-z_{0}\right\|<\delta$, then $\left\|z_{n}^{\prime}-z_{n}\right\|<\left\|x_{n}^{\prime}-x_{n}\right\|+\left\|y_{n}^{\prime}-y_{n}\right\|<\varepsilon_{1}+((1-a) / b) \varepsilon_{1}=\varepsilon$, where $z_{n}=\left(\begin{array}{l}x_{n} \\ y_{n}\end{array}\right) \in o\left(z_{0}\right)$.

Therefore by Definition 3.1 we can see that $o\left(z_{0}\right)$ is stable. The proof is complete.

As an application of Theorem 3.2 we discuss the following example.

Example 3.3.

$$
\begin{gathered}
x(n+1)=f(x(n), y(n)), \\
y(n+1)=g(y(n)),
\end{gathered}
$$

where

$$
x \in \mathbb{R}, y \in \mathbb{R}, \quad f(x, y)=\frac{1}{2} x+10 y, \quad g(y)= \begin{cases}\frac{1}{y}, & y>\frac{1}{3}, \\ \frac{82}{27}-\frac{1}{9} y, & y \leq \frac{1}{3} .\end{cases}
$$

It is easy to verify that system (3.7) has the periodic orbit $\{2,1 / 2\}$. For this periodic orbit, by Theorem 2.2 we have that its corresponding periodic orbit in system (3.6) is the orbit $\{20,30\}$. Moreover by Definition 3.1, we easily prove that the periodic orbit $\{2,1 / 2\}$ is stable, consequently by Theorem 3.2 we have that the periodic orbit $\left\{\left(\begin{array}{c}20 \\ 2\end{array}\right),\left(\begin{array}{c}30 \\ 1 / 2\end{array}\right)\right\}$ of system (3.6) and (3.7) is stable.

\section{Acknowledgment}

This work was partially supported by the Program for New Century Excellent Talents in University (NCET-04-0713).

\section{References}

[1] R. M. Abu-Saris and Q. M. Al-Hassan, On global periodicity of difference equations, Journal of Mathematical Analysis and Applications 283 (2003), no. 2, 468-477.

[2] R. P. Agarwal, M. Meehan, and D. O’Regan, Fixed Point Theory and Applications, Cambridge Tracts in Mathematics, vol. 141, Cambridge University Press, Cambridge, 2001.

[3] L. Alsedà and J. Llibre, Periods for triangular maps, Bulletin of the Australian Mathematical Society 47 (1993), no. 1, 41-53. 
[4] F. M. Atici and G. Sh. Guseinov, Positive periodic solutions for nonlinear difference equations with periodic coefficients, Journal of Mathematical Analysis and Applications 232 (1999), no. 1, 166182.

[5] Y. Chen, All solutions of a class of difference equations are truncated periodic, Applied Mathematics Letters 15 (2002), no. 8, 975-979.

[6] S. Elaydi and R. J. Sacker, Global stability of periodic orbits of non-autonomous difference equations and population biology, Journal of Differential Equations 208 (2005), no. 1, 258-273.

[7] J. Feuer, Periodic solutions of the Lyness max equation, Journal of Mathematical Analysis and Applications 288 (2003), no. 1, 147-160.

[8] J. A. C. Gallas, On the origin of periodicity in dynamical systems, Physica A 283 (2000), no. 1-2, $17-23$.

[9] H. Li and X.-S. Yang, A note on discrete-time dynamical systems under periodic perturbation, Discrete Dynamics in Nature and Society 2006 (2006), Article ID 84697, 5 pages.

[10] H. D. Voulov, On the periodic nature of the solutions of the reciprocal difference equation with maximum, Journal of Mathematical Analysis and Applications 296 (2004), no. 1, 32-43.

[11] Z. Zhou, Periodic orbits on discrete dynamical systems, Computers \& Mathematics with Applications 45 (2003), no. 6-9, 1155-1161.

Huimin Li: Department of Mathematics and Department of Control Science and Engineering, Huazhong University of Science and Technology, Wuhan 430074, China

E-mail address: lihuiminhust@eyou.com

Xiao-Song Yang: Department of Mathematics and Department of Control Science and Engineering, Huazhong University of Science and Technology, Wuhan 430074, China

E-mail address: yangxs@cqupt.edu.cn 


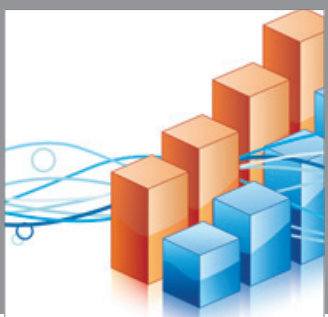

Advances in

Operations Research

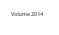

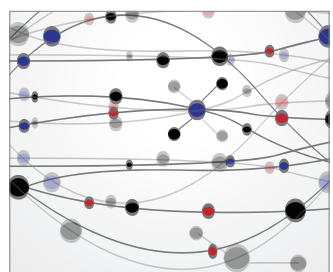

\section{The Scientific} World Journal
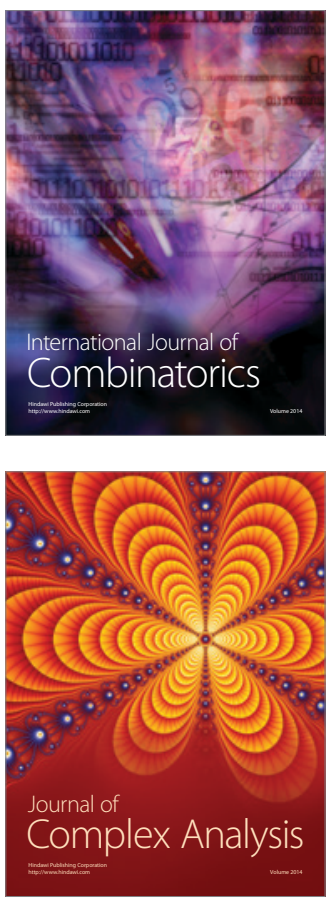

International Journal of

Mathematics and

Mathematical

Sciences
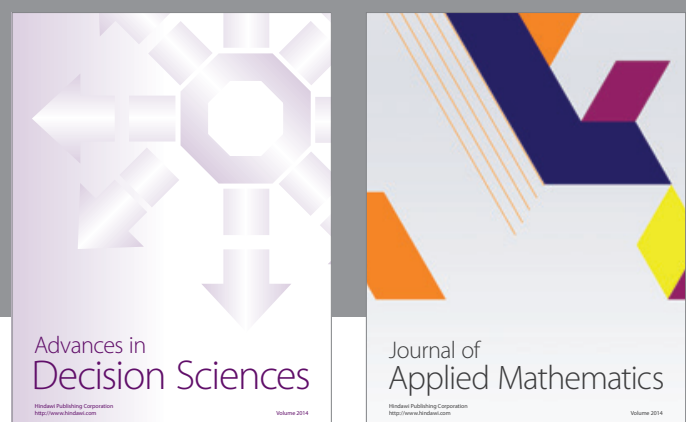

Journal of

Applied Mathematics
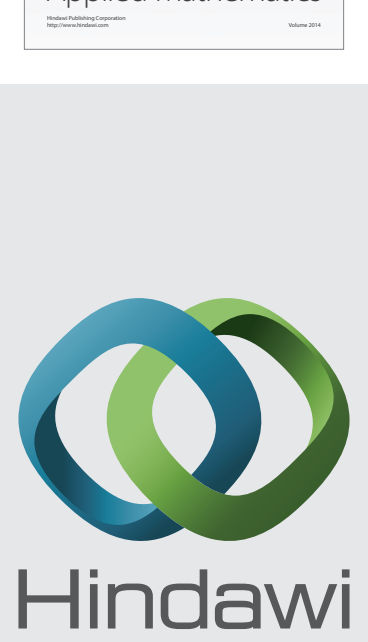

Submit your manuscripts at http://www.hindawi.com
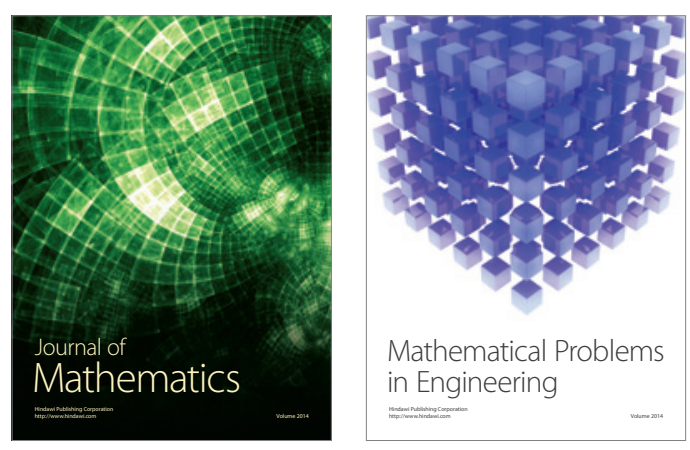

Mathematical Problems in Engineering
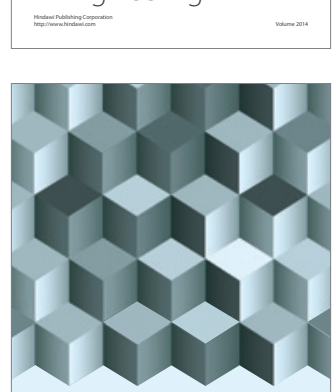

Journal of

Function Spaces
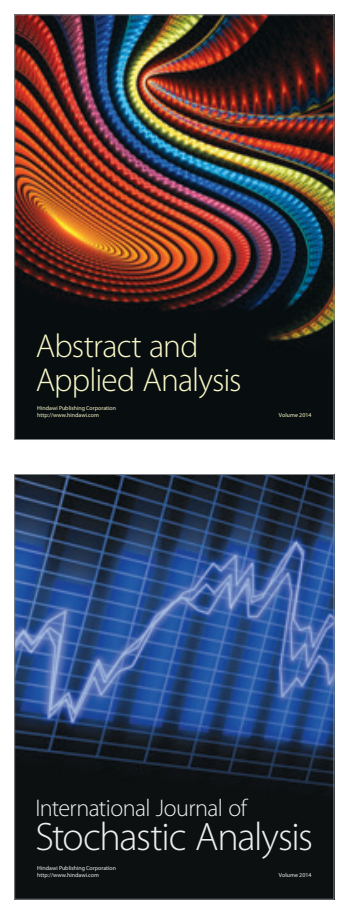

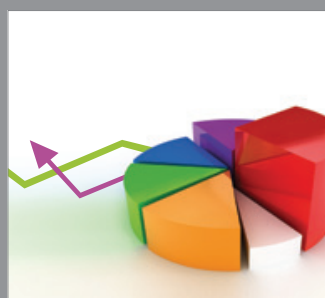

ournal of

Probability and Statistics

Promensencen
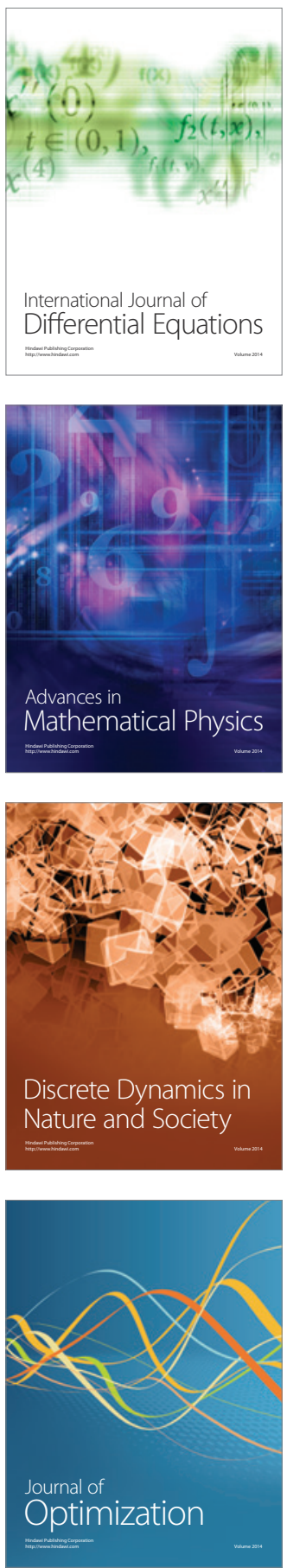